\title{
Possible variability signatures of Supermassive Binary Black Holes in Blazars
}

\author{
Frank M. Rieger \\ Max-Planck-Institut für Kernphysik, 69117 Heidelberg, Germany, \\ E-mail: frank.rieger@mpi-hd.mpg.de
}

\begin{abstract}
Supermassive binary black holes (SBBHs) are considered to be a natural result of hierarchical galaxy mergers, yet phenomenological evidence for the existence of close (separation $\mathrm{d} \leq 0.1 \mathrm{pc}$ ) SBBHs, that are able to coalesce within a Hubble time, still remains ambiguous. Here we show that low-frequency ( $\mathrm{f} \leq 10^{-6} \mathrm{~Hz}$ ) quasi-periodic variability observed from (jet-aligned) radioloud AGNs can provide substantial inductive support for the presence of close SBBHs at their centers: It is argued on physical grounds that such close binary systems are likely to give rise to different (although not independent) periodicities in the radio, optical and $\mathrm{X}$-ray/TeV regime, and, hence that detection of appropriate period ratios significantly corroborates the SBBH interpretation.
\end{abstract}

Workshop on Blazar Variability across the Electromagnetic Spectrum

April 22-25, 2008

Palaiseau, France 


\section{On observational evidence for SBBHs in AGNs}

Supermassive binary black holes (SBBHs) are commonly believed to form during hierarchical galaxy evolution. According to the canonical picture, giant elliptical galaxies (such as the hosts of radio-loud AGNs), are the outcome of merger events between spiral galaxies. Thus if every bright galaxy indeed contains a supermassive black hole (BH), frequent formation of SBBHs is expected. While the existence of wide SBBHs with separations as low as $d \sim 10$ pc [1] appears observationally well grounded today, observational evidence for close $(d \ll 1 \mathrm{pc}) \mathrm{SBBHs}$ is still ambiguous. Dynamical friction and slingshot interaction with stars normally ensure that the binary separation quickly evolves (i.e., in less than $5 \times 10^{8} \mathrm{yr}$ ) to the parsec-scale 泡-包, yet there is still no consensus what (if any) process could allow the binary to overcome its loss cone and to evolve to the sub-parsec scale where gravitational wave emission may be efficiently enough to drive the system to coalescence within a Hubble time. Despite the lack of direct evidence for close binary systems, a number of observational findings (including misalignment, wiggling and precession of jets) has been attributed to the presence of such systems in AGNs, e.g., see [5] for review. Quasiperiodic variability (QPOs) figures most prominently among them and we will thus focus in this paper on that in more details.

\section{On periodic variability as a possible SBBH indicator}

Detailed periodicity analysis, for example, suggests that midterm QPOs with timescales of several tens of days are present in the optical, X-ray and/or TeV lightcurves of blazar sources such as OJ 287, Mkn 421, Mkn 501, 3C 66A and PKS 2155-304. Radio and optical longterm monitoring on the other hand also indicates longterm QPOs with timescales of several years in sources such as BL Lac, ON 231, 3C 273, OJ 287, 3C 345 and AO 0235+16, see Table 1 and Ref. [6] for more details. While tempting, it is nevertheless not the most robust inference to argue for a close SBBH system based on QPOs in just one energy band, as QPOs may be caused by quite a variety of mechanisms, including internal jet rotation, orbiting disk hot spots or specific disk instabilities [7, 8]. However, as we show below, the SBBH case may be substantially strengthened by a cumulative argument that makes reference to several findings, e.g., the observations of QPOs in different energy bands, the apparent divergence in central BH mass estimates [9] and/or the detection of suitable helical jet patterns. In particular, with respect to the latter, there is indeed sufficient evidence for helical motion of components in the pc-scale radio jets of several of the above noted sources, e.g., BL Lac, ON 231, 3C 273, 3C 345 or OJ 287, see [10] for references. Obviously, if this represents a characteristic feature, then the resultant time-dependent change in viewing angle could, via differential Doppler boosting (see below), naturally produce some periodic variability in the observed lightcurves, even for an intrinsically constant flux. In the following, we will discuss some SBBH-related QPO scenarios for blazar-type sources in more details:

- Accretion disk interactions in close SBBHs: Interactions of the companion with the accretion disk around the primary (as well as circumbinary disk interactions) can provide a natural trigger for optical longterm periodicity with periods $P_{\mathrm{obs}}^{\mathrm{opt}}$ of the order of several years [11, 12]. Taking the periodicity to be caused by the secondary $\mathrm{BH}$ crossing the disk around 
the primary $\mathrm{BH}$ on a slightly non-coplanar orbit, implies an upper limit for the intrinsic Keplerian orbital period of the binary of

$$
P_{k} \simeq \frac{2}{(1+z)} P_{\mathrm{obs}}^{\mathrm{opt}}
$$

that is similar to the one inferred under the circumbinary disk assumption. Of course, some deviations from strict periodicity may well be expected due to, for example, internal disc warping or precession.

- Differential Doppler boosting along helical jet paths: Helical jet paths can naturally arise in binary systems where (at least) one of the BH produces a collimated jet (see below). Suppose that an emitting component follows such a path. Differential Doppler boosting may then lead to spectral flux modulation

$$
S(v, t)=\delta(t)^{3+\alpha} S^{\prime}(v),
$$

where $S^{\prime}$ is the flux measured in the comoving frame and $\delta(t)=1 /\left(\gamma_{b}\left[1-\beta_{b}(t) \cos \theta(t)\right]\right)$ is the time-dependent Doppler factor, with $\theta(t)$ the actual angle between the component's velocity and the line-of-sight, and $\gamma_{b} \sim 10$ a typical blazar bulk Lorentz factor. A periodically changing viewing angle due to quasi-regular helical motion thus naturally leads to a periodically changing Doppler factor and thus to a possible periodicity in the observed flux [10].

- Shortening effects for observed QPOs: If the helical jet appearance is caused by nonballistic helical motion (whether orbital SBBH or precession-driven), observable periods may appear strongly shortened with respect to their real physical driving periods $P$ according to [10]

$$
P_{\mathrm{obs}} \simeq(1+z) \frac{P}{\gamma_{b}^{2}} .
$$

This is based on the fact that observationally we measure arrival times of pulses, say emitted at $\mathrm{A}$ and $\mathrm{B}$, where the velocity vector of the emitting knot points closest towards us, whereas - due to the relativistic motion of the knot at small inclinations - the light travel distance for a pulse emitted at B is much smaller than for a pulse emitted at A.

Many scenarios for the origin of helical AGN jets assume a close SBBH system as central driving source (but see also below). In the SBBH framework, we may distinguish at least two different scenarios:

- Orbital-driven helical motion: Orbital motion of a jet-emitting BH can lead to the appearance of helical jet paths on small scales. As the high energy emission in radio-loud AGNs is usually thought to be produced on smaller jet scales, the optical, X-ray and/or TeV flux may well show signs of orbital modulation. Taking the optical (longterm) periodicities to arise due to disk interactions, for example, generally implies close SBBHs with separations $d \sim 10^{16}-10^{17} \mathrm{~cm}$ and characteristic Keplerian orbital periods $P_{k}$ of the order of several 
years. This suggests that the jet-dominated high energy emission may show quasi-periodic modulation with characteristic periods of

$$
P_{\mathrm{obs}} \sim 30\left(\frac{P_{\mathrm{obs}}^{\mathrm{opt}}}{10 \mathrm{yr}}\right)\left(\frac{15}{\gamma_{b}}\right)^{2} \text { days },
$$

provided shortening effects are fully taken into account, see Eq. (2.3).

- Precession-driven helical motion: In most cases, geodetic or gravito-magnetic relativistic effects due to the gravitational field or the motion of the companion are too slow $\left(P \geq 10^{4} \mathrm{yr}\right)$ to be of interest. Much more interesting is a Newtonian-type jet precession that may arise, for example, due to tidally induced perturbations in the disk around the jet-emitting primary [13, 14]. If the inner disk is thick enough (Mach number $M \leq 1 / \alpha$ ), such perturbations can lead to a rigid-body precession of its innermost parts. The associated driving period $P_{p}$ is typically about an order of magnitude larger than the orbital period $P_{k}$ [10]. If, as commonly believed, the radio emission emerges on larger jet scales, it may thus be primarily modulated by Newtonian jet precession. This would result in observable periods

$$
P_{\mathrm{obs}}^{\mathrm{radio}} \sim 2\left(\frac{P_{\mathrm{obs}}^{\mathrm{opt}}}{10 \mathrm{yr}}\right)\left(\frac{10}{\gamma_{b}}\right)^{2} \mathrm{yr},
$$

comparable to those observed (cf. Table 1).

In principle, helical jet paths and QPOs might also arise in scenarios that do not require the presence of a supermassive binary system, and in the following we will thus shortly comment on two of them: (i) In the lighthouse jet model [15], helical motion of components and short-term QPOs are caused by internal jet rotation. Yet, in most cases the inferred QPO periods are too small to account for midterm periodicity [10] and the model itself cannot account for optical longterm QPOs. Of course, this does not exclude the possibility that the jet emitted by a BH in a binary system is internally rotating. (ii) Spin-induced disk precession has been proposed for the $\simeq 106 \mathrm{~d}$ radio periodicity observed in Sgr A* [16] and later applied to the AGN context [17], suggesting that jet precession with periods as low as $P_{p} \sim 10 \mathrm{yr}$ might be possible. Yet, while interesting, the results very much depend on less-constrained disk properties and the question whether propagating bending waves are indeed able to establish quasi-rigid body precession on such timescales [18]. In addition, such a scenario by itself also does not provide a simple explanation for different periods.

\section{Applications}

For an illustration of SBBH considerations, let us consider the following candidate sources:

- BL Lac Mkn 501: During the 1997 high state Mkn 501 showed evidence for a 23d-periodicity in its TeV and X-ray light curves [19], suggestive of a close SBBH system with orbital period of $P_{k} \sim(6-14) \mathrm{yr}$ at its center [20]. Although some midterm QPOs could possibly also arise due to disk instabilities [8], evidence for optical longterm QPOs with period of $\simeq 10$ yr [21] adds further support to the SBBH interpretation. Additional support seems to come 


\begin{tabular}{|c|c|c|c|c|c|c|c|}
\hline name & redshift $z$ & periods $P_{\mathrm{obs}}$ & Ref. & $(m+M) / 10^{8} M_{\odot}$ & $P_{k}[\mathrm{yr}]$ & $d / 10^{16} \mathrm{~cm}$ & $\tau_{g} / 10^{8} \mathrm{yr}$ \\
\hline Mkn 501 & 0.034 & $\begin{array}{l}23.6 \mathrm{~d} \text { (X-ray) } \\
\sim 23 \mathrm{~d}(\mathrm{TeV}) \\
10.06 \mathrm{yr} \text { (optical) }\end{array}$ & |19 & $(2-7)$ & $(6-14)$ & $(2.5-6)$ & $\leq 5.5$ \\
\hline BL Lac & 0.069 & $\begin{array}{l}13.97 \mathrm{yr} \text { (optical) } \\
\sim 4 \mathrm{yr} \text { (radio) }\end{array}$ & 22 & $(2-4)$ & $(13-26.1)$ & $(4.8-9.7)$ & $\leq 29$ \\
\hline $3 C 273$ & 0.158 & $\begin{array}{l}13.65 \mathrm{yr} \text { (optical) } \\
8.55 \mathrm{yr} \text { (radio) }\end{array}$ & 24 & $(6-10)$ & $(11.8-23.5)$ & $(6.5-12)$ & $\leq 3.5$ \\
\hline OJ 287 & 0.306 & $\begin{array}{l}11.86 \mathrm{yr} \text { (optical) } \\
\sim 12 \mathrm{yr} \text { (infrared) } \\
\sim 1.66 \mathrm{yr} \text { (radio) } \\
\sim 40 \mathrm{~d} \text { (optical) }\end{array}$ & 28 & 6.2 & $(9.1-18.2)$ & $(5.5-8.8)$ & $\leq 1.7$ \\
\hline $3 \mathrm{C} 66 \mathrm{~A}$ & 0.444 & $\begin{array}{l}4.52 \mathrm{yr} \text { (optical) } \\
65 \mathrm{~d} \text { (optical) }\end{array}$ & (29) & $\geq 1$ & $(3.1-6.3)$ & $\geq 1.5$ & 2.08 \\
\hline $0235+16$ & 0.940 & $\begin{array}{l}2.95 \mathrm{yr} \text { (optical)? } \\
8.2 \mathrm{yr} \text { (optical)? } \\
5.7 \mathrm{yr} \text { (radio) }\end{array}$ & (29) & $\geq 1$ & $(1.5-3.1)$ & $\geq 0.95$ & $\leq 0.3$ \\
\hline
\end{tabular}

Table 1: SBBH properties for a sample of blazar candidate sources based on reported QPO evidence (see Ref., column 4). Abbreviations are: Total mass $(m+M)$, Keplerian period $P_{k}$, inferred separation $d$ and associated gravitational life time $\tau_{\text {grav }}$ for mass ratios $m / M \geq 0.01$.

from the apparent disagreement in BH mass estimates inferred from host galaxy and high energy observations. These divergent estimates can be reconciled if the jet dominating the VHE emission is produced by the less massive BH in a binary system [?].

- BL Lac OJ 287: Optical monitoring indicates a $\sim 12$ yr longterm [11] and a possible $\sim 40 \mathrm{~d}$ midterm periodicity [28]. Assuming the longterm periodicity to be caused by disk interactions suggests a Keplerian orbital period of $P_{k} \simeq(9-18)$ yr. Orbital driven helical jet motion then implies an observable midterm periodicity $P_{\mathrm{obs}} \sim(40-80)\left(10 / \gamma_{b}\right)^{2}$ d. Radio emission, emerging from larger, Newtonian precession modulated scales may then show QPOs with $P_{\mathrm{obs}} \sim(1-2) \mathrm{yr}$, as indeed indicated in the radio data [27]. This suggests that a close SBBH system might offer a powerful explanatory framework. (Note that recent observations of a possible $20 \mathrm{~d}$ decrease in the longterm period have been interpreted as due to the loss of orbital energy by gravitational wave emission in a highly eccentric, very massive $2 \times 10^{10} M_{\odot}$ binary [33]. Yet, given all uncertainties, this conclusion seems less robust and certainly needs further confirmation.)

In Table 1, SBBH properties have been derived for a sample of blazar candidate sources using the observed longterm QPOs as input (in all cases except for Mkn 501).

\section{Implications}

The above considerations support the hypothesis that close SBBHs may be present in (at least) some radio-loud AGNs. This indicates that further radio, optical and VHE monitoring and advanced QPO analysis can provide an important diagnostic tool to unveil the nature of the central engine in AGNs. The analysis of our source candidates suggests the following:

(1) According to the last column in Table 1, there is strong phenomenological evidence for SBBH 
lifetimes $\tau_{\text {grav }} \ll$ Hubble time $t_{H} \sim 10^{10}$ yr. This supports the notion that SBBHs in AGNs can indeed overcome their evolutionary "bottleneck" and coalesce within cosmic times as assumed in hierarchical galaxy formation (merger) models.

(2) Disk interactions can provide a natural trigger for enhanced jet activity and may lead to recurrent ejection of superluminal components and a hot disk outflow with luminosity up to $L_{\text {edd }}$ of the secondary. The delay for enhanced jet emission depends on the disc properties, but may be sufficiently small (e.g., less than two years) if accretion occurs in the advection-dominated regime. (3) The characteristic binary separations $d$ (derived assuming disk crossing by the secondary) are consistent with the maximum disk size $r_{d} \sim\left(10^{3}-10^{4}\right) r_{g}$ for an (initially) stable standard SS disk around the primary.

\section{Conclusions}

While QPO detection in a single energy band may hint to the presence of close SBBHs in blazars, observations of helical jet paths, different periods in different energy bands and/or apparent central BH mass disagreements can give decisive weight to such an interpretation.

\section{References}

[1] C. Rodriguez et al., ApJ 646 (2006), 49

[2] M.C. Begelman, R.D. Blandford, M.J. Rees, Nature 287 (1980), 307

[3] Q. Yu, MNRAS 331(2002), 935

[4] D. Merritt, M. Milosavljević, Living Reviews in Relativity 8 (2005), 8

[5] S. Komossa, Mem. S.A.It. 77 (2006), 733

[6] F.M. Rieger, Ap\&SS 309 (2007), 271

[7] F.K. Liu, G. Zhao, X-B. Wu, ApJ 650 (2006), 749

[8] J.H. Fan, F.M. Rieger, T.X. Hua et al., APh 28 (2008), 508

[9] F.M. Rieger, K. Mannheim, A\&A 397 (2003), 121

[10] F.M. Rieger, ApJL 615 (2004), L5

[11] A. Sillanpää, S. Haarala, M.J. Valtonen et al., ApJ 325 (1988), 628

[12] K. Hayasaki, S. Mineshige, H. Sudou, PASJ 59 (2007), 427

[13] J. Katz, ApJ 478 (1997), 527

[14] J. Larwood, MNRAS 299 (1998), L29

[15] M.Camenzind, M. Krockenberger, A\&A 255 (1992), 59

[16] S. Liu, F. Melia, ApJ 573 (2002), L23

[17] A. Caproni et al., ApJ 616 (2004), L99

[18] R.P. Nelson, J.C.B. Papaloizou, MNRAS 315 (2000), 570

[19] S. Osone, APh 26 (2006), 209 
[20] F.M. Rieger, K. Mannheim, A\&A 359 (2000), 948

[21] Y.H. Yang et al., AcASn 41 (2007), 407

[22] J.H. Fan et al., ApJ 507 (1998), 173

[23] B.C. Kelly et al., ApJ 591 (2003), 695

[24] J.H. Fan et al., ChA\&A 25 (2001), 282

[25] A. Ciaramella et al., A\&A 419 (2004), 485

[26] J.H. Fan et al., A\&AS 133 (1998), 163

[27] P.A. Hughes et al., ApJ 503 (1998), 662

[28] J. Wu et al., AJ 132 (2006), 1256

[29] J.H. Fan et al., A\&A 381 (2002), 1

[30] M. Lainela et al. ApJ 521 (1999), 561

[31] C. Raiteri et al., A\&A 459 (2006), 731

[32] C. Raiteri et al., A\&A 377 (2001), 396

[33] M.J. Valtonen et al., Nature 452 (2008), 851 